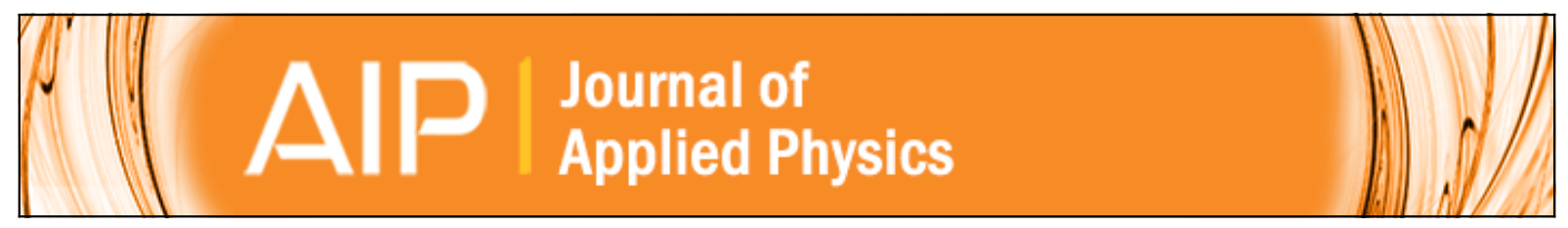

\title{
Schlieren technique applied to the arc temperature measurement in a high energy density cutting torch
}

L. Prevosto, G. Artana, B. Mancinelli, and H. Kelly

Citation: Journal of Applied Physics 107, 023304 (2010); doi: 10.1063/1.3291099

View online: http://dx.doi.org/10.1063/1.3291099

View Table of Contents: http://scitation.aip.org/content/aip/journal/jap/107/2?ver=pdfcov

Published by the AIP Publishing

\section{Articles you may be interested in}

Numerical investigation of the double-arcing phenomenon in a cutting arc torch

J. Appl. Phys. 116, 023301 (2014); 10.1063/1.4887490

Departures from local thermodynamic equilibrium in cutting arc plasmas derived from electron and gas density measurements using a two-wavelength quantitative Schlieren technique

J. Appl. Phys. 109, 063302 (2011); 10.1063/1.3552304

Measurement of the electron density in a microwave plasma torch at atmospheric pressure

Appl. Phys. Lett. 95, 201502 (2009); 10.1063/1.3266923

On the physical origin of the nozzle characteristic and its connection with the double-arcing phenomenon in a cutting torch

J. Appl. Phys. 105, 013309 (2009); 10.1063/1.3041636

Temperature measurement of an atmospheric-pressure plasma torch

Rev. Sci. Instrum. 70, 3032 (1999); 10.1063/1.1149864

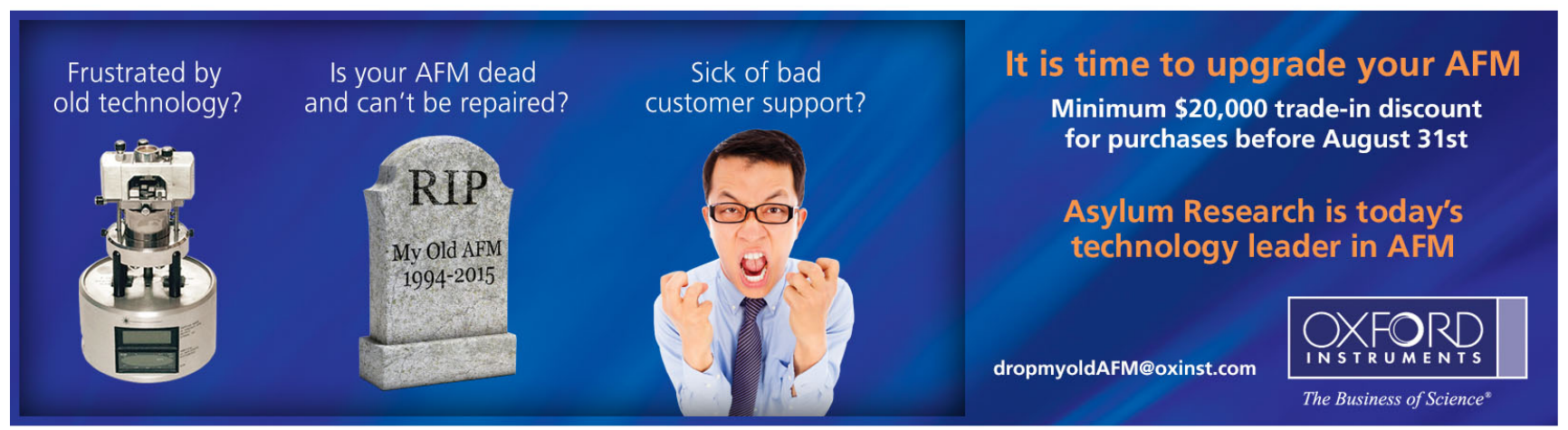




\title{
Schlieren technique applied to the arc temperature measurement in a high energy density cutting torch
}

\author{
L. Prevosto, ${ }^{1, a)}$ G. Artana, ${ }^{2}$ B. Mancinelli, ${ }^{1}$ and H. Kelly ${ }^{3}$ \\ ${ }^{1}$ Departamento Ing. Electromecánica, Grupo de Descargas Eléctricas, Universidad Tecnológica Nacional, \\ Regional Venado Tuerto, Las Heras 644, Venado Tuerto, Santa Fe 2600, Argentina \\ ${ }^{2}$ Departamento Ing. Mecánica, Laboratorio de Fluidodinámica, Facultad de Ingeniería (UBA), Paseo Colon \\ 850 (C1063ACV), Buenos Aires, Argentina \\ ${ }^{3}$ Departamento de Física, Instituto de Física del Plasma (CONICET), Facultad de Ciencias Exactas y \\ Naturales (UBA), Ciudad Universitaria Pab. I, Buenos Aires 1428, Argentina
}

(Received 30 September 2009; accepted 14 December 2009; published online 25 January 2010)

\begin{abstract}
Plasma temperature and radial density profiles of the plasma species in a high energy density cutting arc have been obtained by using a quantitative schlieren technique. A Z-type two-mirror schlieren system was used in this research. Due to its great sensibility such technique allows measuring plasma composition and temperature from the arc axis to the surrounding medium by processing the gray-level contrast values of digital schlieren images recorded at the observation plane for a given position of a transverse knife located at the exit focal plane of the system. The technique has provided a good visualization of the plasma flow emerging from the nozzle and its interactions with the surrounding medium and the anode. The obtained temperature values are in good agreement with those values previously obtained by the authors on the same torch using Langmuir probes.
\end{abstract}

(C) 2010 American Institute of Physics. [doi:10.1063/1.3291099]

\section{INTRODUCTION}

Plasma cutting is a process of metal cutting at atmospheric pressure by an arc plasma jet, where a transferred arc is generated between a cathode and a work piece (the metal to be cut) acting as the anode. A high-quality cut requires a narrow, hot, and high-velocity plasma jet, i.e., a high energy density arc jet. To this end, a new generation of cutting torches, the so-called "high energy density torch" has been developed. These torches are characterized by an arc current intensity in the range of 30-100 A, flat cathodes, oxygen as the plasma gas, very small nozzle diameters $(\approx 1 \mathrm{~mm})$, and by the generation of an underexpanded supersonic arc jet with a shock wave close to the nozzle exit. ${ }^{1-4}$

Optical methods represent a versatile tool for performing nonintrusive, quantitative measurements in transparent media. In particular, refractive techniques allow the investigation of the temperature distribution in transparent flows by measuring its index of refraction (or its spatial derivatives). These refractive techniques can be divided into two groups: the interference methods, which are based on the difference in length of the light ray paths, and the methods based on the angular deflections of the light rays, such as shadowgraph and schlieren. The schlieren technique is based on the angular deflection undergone by a light ray when passing through a region characterized by refractive index inhomogeneities. In fluids, these inhomogeneities are generally caused by density or temperature variations; therefore, the measured optical data can be processed in order to gain information on these fluid variables. Among the optical methods suitable for evaluating, for instance, temperature fields, the schlieren technique is attractive for its simplicity, ease of application,

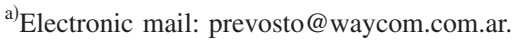

low cost, and accuracy of the results. Typical schlieren systems include a pointlike light source, usually a xenon flash lamp. The light from the source is collimated by a lens, which is at least as large as the object that is being analyzed. After the collimated beam passes through the test section, it is focused by a second lens. A sharp edge (usually a knife edge) is then placed at the exit focal point of the system. When the knife edge is placed, let us say, horizontally, a light ray that is deflected upwards by positive vertical gradients passes freely to the light detector, whereas a ray deflected downwards by negative vertical gradients is blocked out by the knife. Hence, positive vertical density gradients are visualized as light areas and negative vertical gradients as dark ones. An extensive description of the schlieren technique can be found elsewhere. ${ }^{5}$ This technique was primarily conceived as a visualization (qualitative) technique and still only a few percent of schlieren publications involves quantitative evaluations (i.e., the measurement of gas density or temperature ${ }^{6}$ - and also gas velocity ${ }^{7}$-from schlieren images) in spite of the fact that digital image processing software simplify such evaluations.

The available published works on cutting arc visualization techniques are scarce and were summarized in a recent review. ${ }^{8}$ The published papers on the subject are those by Bemis and Settles. ${ }^{9,10}$ A study of the fluid dynamic and plasma dynamic mechanism involved in the formation of low-speed and high-speed dross was carried out through the visualization of the plasma cutting front. ${ }^{9}$ A commercial $30 \mathrm{~A}$ oxygen plasma cutting torch was used for the cutting $2.9 \mathrm{~mm}$ thick mild-steel plates. Schlieren imaging was used to reveal the flows associated with the shielding gas and the rapid mixing of the plasma jet with the gaseous atmosphere underneath the work piece. Ultraviolet (UV) photography revealed 


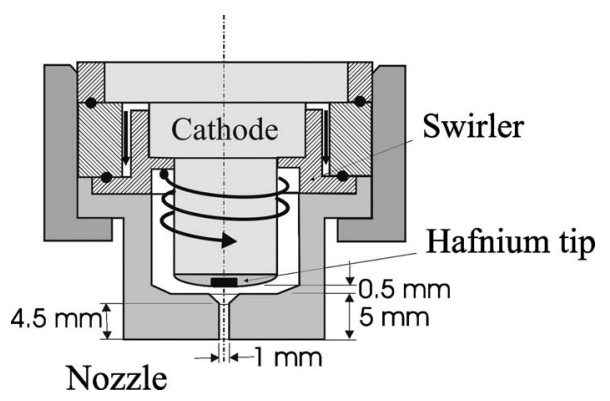

FIG. 1. Schematic of the arc torch indicating several geometric dimensions.

that the electric arc attaches at the kerf bottom leading edge, where an anode spot forms and radiates strongly in the UV due to oxygen and iron ions. ${ }^{10}$

In this work, a quantitative interpretation of the schlieren technique applied to a high energy density cutting arc is for the first time reported. The technique allows measuring plasma composition and temperature by processing the graylevel contrast values of a digital schlieren image recorded at the observation plane for a given position of a transverse knife located at the exit focal plane of the optical system. Also the technique provides a good visualization of the plasma flow emerging from the nozzle and its interactions with the surrounding medium and the anode.

The organization of the paper is as follows. In Sec. II, the experimental arrangement are presented. The procedure of the quantitative interpretation of the schlieren images and its results are presented and discussed in Sec. III and the conclusions are presented in Sec. IV.

\section{EXPERIMENTAL ARRANGEMENT}

The high energy density cutting torch used in this study consisted of a cathode centered above an orifice in a converging-straight copper nozzle. The cathode was made of copper ( $7 \mathrm{~mm}$ in diameter) with a hafnium tip $(1.5 \mathrm{~mm}$ in diameter) inserted at the cathode center. A flow of oxygen gas cooled the cathode and the nozzle and was also employed as the plasma gas. The gas passed through a swirl ring to provide arc stability. The nozzle consisted in a converging-straight bore (with a converging length of $1 \mathrm{~mm}$ and a bore $1 \mathrm{~mm}$ in diameter, $4.5 \mathrm{~mm}$ length) in a copper holder surrounding the cathode (with a separation of $0.5 \mathrm{~mm}$ between the holder and the cathode surface). To avoid plasma contamination by metal vapors from the anode (usually the work piece to be cut), a rotating steel disk with 200 $\mathrm{mm}$ in diameter and $15 \mathrm{~mm}$ thickness was used as the anode. ${ }^{11}$ In this study, the disk upper surface was located at 8 $\mathrm{mm}$ from the nozzle exit. The arc was transferred to the edge of the disk, and the rotating frequency of the disk was equal to $29.5 \mathrm{~Hz}$. At this velocity, a well-stabilized arc column was obtained, and no noticeable damage in the lateral surface of the anode disk was found. Thus, practically no metal vapors from the anode were present in the arc. A scheme of the torch indicating several geometric dimensions is presented in Fig. 1. By performing a small orifice (1 $\mathrm{mm}$ in diameter) on the

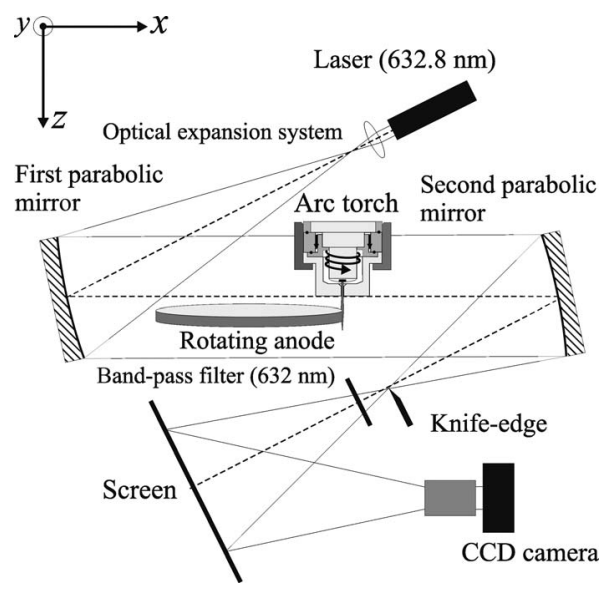

FIG. 2. Schematic of the Z-type two-mirror schlieren system.

cathode lateral surface the pressure in the plenum chamber $(0.8 \mathrm{MPa})$ was measured by connecting a pressure meter at the upper head of the cathode.

A Z-type two-mirror schlieren system was used in this research. ${ }^{5}$ The system included a $20 \mathrm{~mW}$ continuous laser with a main wavelength of $632.8 \mathrm{~nm}$ as the light source. The laser beam was expanded to a diameter of $50 \mathrm{~mm}$ at the first mirror surface by using an optical expansion system. There were two parabolic mirrors (100 $\mathrm{mm}$ diameter) with focal distances of $f_{1}=900$ and $f_{2}=1100 \mathrm{~mm}$ for the first and second mirror, respectively, and a knife edge with $x-z$ movement that could be accurately controlled by a two-dimensional micrometer. The first mirror serves to collimate the incoming light, while the second mirror forms an image of the observation region (nozzle exit, cutting arc, and lateral surface of the anode in this case) onto a white screen. Figure 2 shows a schematic of the experimental set-up. The cutting torch was positioned vertically with the streamwise direction of the plasma flow along the $z$ direction and the knife edge was positioned parallel to this direction to provide the best sensibility perpendicular to the arc axis. A band-pass filter centered at the laser wavelength was used to block the plasma light emitted from the arc. The schlieren images were acquired with a charge-coupled device Lumenera digital camera, which can provide 30 frames per second at a spatial resolution of $640 \times 480$ pixels. These images were stored in BMP format and digitized by an 8-bit gray-level frame grabber.

\section{EXPERIMENTAL RESULTS AND DISCUSSION}

Schlieren visualization of arc plasmas is based on the fact that the arc represents a transparent medium for a laser light having an appropriate wavelength. As for every optical medium, the refractivity $(n-1$, being $n$ the refraction index of the medium) is the characteristic parameter. Since $n$ is related to the plasma composition (that in turn depends on the plasma temperature), the measurement of the arc plasma refractivity leads to conclusions on that plasma parameters. The plasma refractivity can be described as a sum of the individual refractivities of the different species, so it is composed of the contributions due to neutrals, ions, and electrons ${ }^{12}$ 


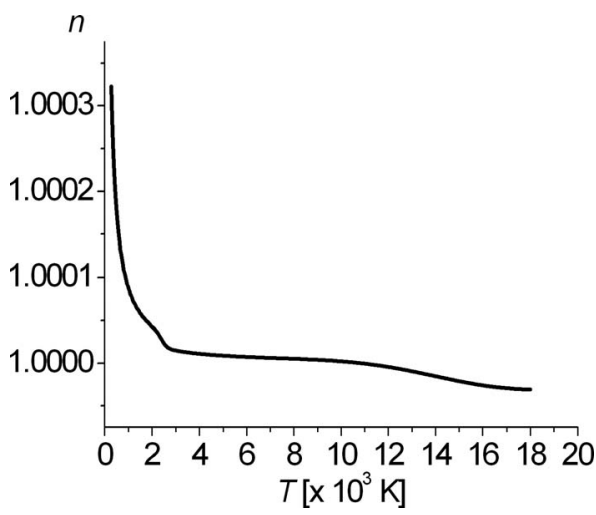

FIG. 3. Refractive index of atmospheric pressure oxygen plasma as a function of the temperature, calculated under a LTE.

$$
n-1=(n-1)_{n}+(n-1)_{+}+(n-1)_{e} .
$$

Each individual refractivity contribution can be written as

$$
(n-1)_{e}=-\frac{1}{4 \pi \varepsilon_{0}} \frac{e^{2} \lambda^{2}}{2 \pi m_{e} c_{0}^{2}} n_{e},
$$

for the electrons and

$$
(n-1)_{+, n}=\frac{1}{4 \pi \varepsilon_{0}} 2 \alpha_{+, n}(\lambda) n_{+, n},
$$

for the ions and neutrals. In Eqs. (2) and (3) $e$ is the electronic charge, $m_{e}$ is the electron mass, $c_{0}$ is the light velocity, and $\lambda$ is the wavelength of the light used for probing the plasma. $\alpha_{+, n}$ is the polarizability of ions and neutrals, respectively, dependent on the light wavelength, $n_{e}, n_{+}$, and $n_{n}$, are the densities of electrons, ions, and neutrals, respectively, and $\varepsilon_{0}$ is the free space permittivity. The ion refractivity can in general be neglected as compared with the electron refractivity since both densities are the same. On the other hand, the neutral refractivity cannot be neglected because as will be shown later there are several zones in which the neutral density is by far larger than the electron density. Equations (2) and (3) are valid for electromagnetic waves with frequencies $w \gg w_{p}$, where $\omega_{p} \equiv \sqrt{n_{e} e^{2} /\left(\varepsilon_{0} m_{e}\right)}$ is the plasma frequency. ${ }^{13}$ For the experimental conditions considered here (thermal plasmas at temperatures of the order of $1 \mathrm{eV}$ and pressures around the atmospheric value, leading to plasma densities of about $10^{23} \mathrm{~m}^{-3}$ ) it results $\omega_{p}=10^{13} \mathrm{~s}^{-1}$, a value much smaller than the employed optical frequency ( $\approx 10^{15} \mathrm{~s}^{-1}$ ). Replacing Eqs. (2) and (3) in Eq. (1), and using the expression of the plasma frequency, the refractive index for a partially ionized plasma is given by

$$
n=1-\frac{1}{2}\left(\frac{\omega_{p}}{\omega}\right)^{2}+\frac{1}{4 \pi \varepsilon_{0}} 2 \alpha_{n} n_{n}
$$

The refractive index for atmospheric pressure oxygen plasma, calculated under a local thermal equilibrium assumption (LTE) (Ref. 14) results a function of the plasma temperature $(T)$, and is shown in Fig. 3. In this computation, the neutral contribution to the oxygen refractive index was taken into account using the Gladstone-Dale constant's ${ }^{5}$ assuming that the polarizability of the oxygen atoms and the oxygen molecules are similar. For high temperature values the electron contribution to the refractive index is dominant, while in the opposite case (low temperature values) the neutral contribution is the relevant one. For a temperature value around $10 \mathrm{kK}$ (i.e., when the ionization degree is only $\approx 1 \%$ ) both contributions are equivalent.

It is well known that when a light ray passes through an inhomogeneous medium, it suffers a deviation in its trajectory by a certain angle $\varepsilon$. This angle depends both on the refractive index and on the thickness of the medium under test. The equation of the ray path through an inhomogeneous medium is expressed as ${ }^{5}$

$$
\varepsilon_{\xi}=\int \frac{1}{n} \frac{d n}{d \xi} d y,
$$

where $y$ is the optical axis direction and $\xi$ can be the $x$ or $z$ coordinate, depending on the direction in which the knife blocks out the light. Since that in this work the knife edge was positioned parallel to the $z$ direction, the analysis was done for the $x$ direction. By considering the approximation of small $\varepsilon$, the measured deviation at the observation plane is $\delta_{x}=f_{2} \tan \varepsilon \approx f_{2} \varepsilon$. Then, Eq. (5) can be written as

$$
\delta_{x}(x)=f_{2} \int \frac{1}{n} \frac{\partial n}{\partial x} d y .
$$

Since the arc column presents cylindrical symmetry [i.e., $n$ $=n(r, z)]$, Eq. (6) can be expressed as

$$
\delta_{x}(x)=2 f_{2} \int_{0}^{\infty} \frac{1}{n} \frac{\partial n}{\partial r} \frac{x}{r} d y
$$

where $r$ is the radial coordinate measured from the arc axis.

Ray path deviations $\delta_{x}$ can alter the local illuminance on the screen only if the deviations are weak enough so as the entire image is not moved onto, or off of, the knife edge (i.e., when the measuring range of the schlieren system has not been exceeded). ${ }^{5}$ When this condition is satisfied, the contrast $C$ of the light pattern on the screen is the output of the schlieren system. Contrast in the schlieren image is a quantity defined as the ratio of the differential illuminance at a given image pixel to the value of its background level illuminance (a reference schlieren image registered without the presence of the tested medium but with the knife edge at its reference position). $C$ is related with the ray path deviations as $C(x)=\delta(x) / a$ where $a$ represents the height of the image (measured from the knife edge and without the presence of the tested medium) that remains unobstructed by the knife edge. ${ }^{5}$ Then Eq. (7) can be written as

$$
C(x)=2 \frac{f_{2}}{a} \int_{0}^{\infty} \frac{1}{n} \frac{\partial n}{\partial r} \frac{x}{r} d y \approx 2 \frac{f_{2}}{a n_{\infty}} \int_{0}^{\infty} \frac{\partial n}{\partial r} \frac{x}{r} d y .
$$

where the last approximation in Eq. (8) follows from the fact that $n$ is quite close to the refraction index of the surrounding medium $n_{\infty}$ (see Fig. 3). The differential illuminance for each pixel of the image was obtained by subtracting from the digital schlieren image in the presence of the plasma flow, the background reference schlieren image. The contrast distribution in the image was obtained dividing at each pixel point the differential illuminance by the intensity of illumi- 


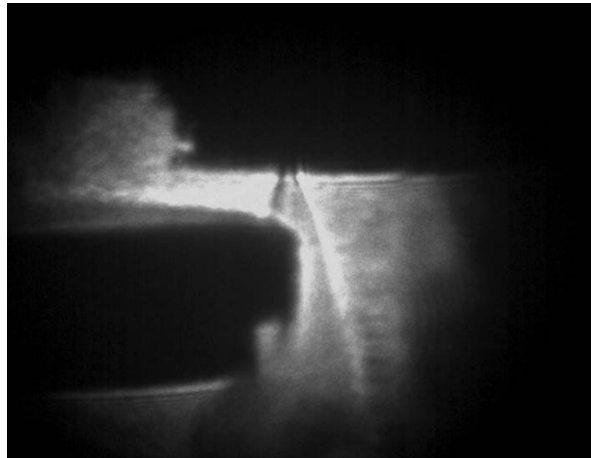

FIG. 4. Typical gray-scale schlieren image of the 30 A high energy density cutting arc. The percentage of the knife edge cutoff was $\approx 50 \%$ (blocking on the left side of the laser beam).

nance of the reference image at the corresponding pixel.

A typical $30 \mathrm{~A}$ arc schlieren image is shown in Fig. 4. In this case, the knife edge was located so as to cut $\approx 50 \%$ of the unperturbed perpendicular beam. This knife edge location provides a great sensibility to the radial gradients of the refractive index and does not exceed the measuring range of the system. As it can be seen, a good visualization of the plasma flow structure and its interaction with the surrounding medium and the anode appears in the picture. Since the knife edge was placed vertically (cutting on the left side of the image), positive plasma density radial gradients (directed to the image right side) are visualized as light areas and negative gradients as dark ones.

By using the Abel inversion technique, ${ }^{15} \mathrm{Eq}$. (8) can be inverted to obtain the radial refractivity gradient. Then, using Eq. (4) and the equation of state (assuming that the arc pressure is uniform along the radius), and assuming also that LTE is accomplished (so the gas dissociation and ionization can be evaluated using Saha equation), ${ }^{14}$ the system becomes closed and hence radial profiles of the plasma temperature and composition can be derived.

To invert the Eq. (8), the plasma flow was subdivided into $N$ differential concentric annulus (see Fig. 5). In order to obtain a good behavior for the solutions it was found that $N=10$ was high enough to obtain smooth radial profiles of the unknowns. Similar values of $N$ have been successfully

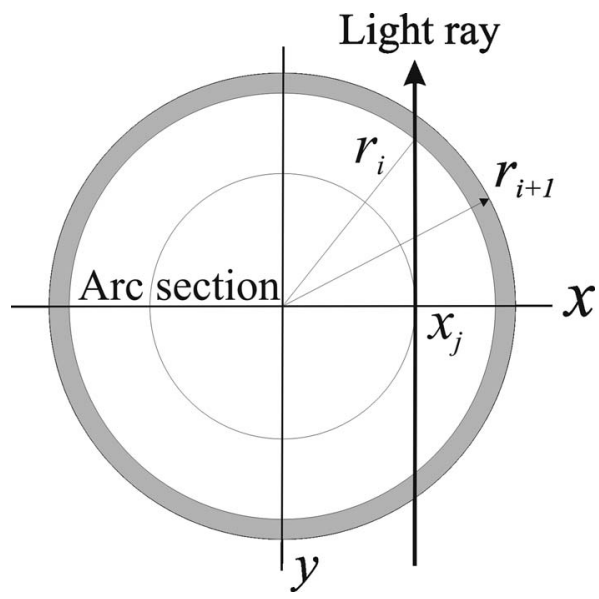

FIG. 5. Scheme of the arc section showing the radial discretization used to invert the Eq. (8).

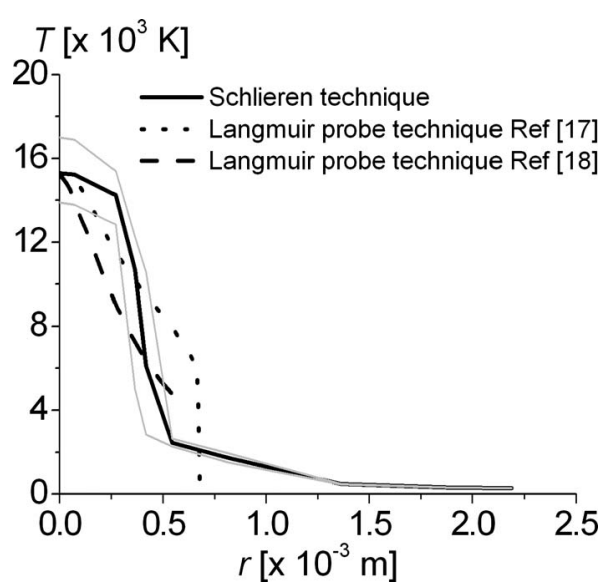

FIG. 6. Plasma temperature radial profile obtained at $z=3.5 \mathrm{~mm}$ from the nozzle exit. The gray thin lines indicate the limits of the uncertainty range in the $T$ values. The plasma temperature profile previously obtained by the authors using Langmuir probes is also shown.

used in inversions of physical quantities. ${ }^{15}$ Then, a discretization of the integral in Eq. (8) into a set of $N$ linear equations was performed

$$
\begin{aligned}
C\left(x_{j}\right) & \left.=\frac{2 f_{2}}{a n_{\infty}} \sum_{i \geq j}^{N-1} \frac{\partial n}{\partial r}\right)_{j} x_{j} \int_{\sqrt{r_{i}-x_{j}}}^{\sqrt{r_{i+1}-x_{j}}} \frac{d y}{\sqrt{x_{j}^{2}+y^{2}}} \\
& \left.=\frac{2 f_{2}}{a n_{\infty}} \sum_{i \geq j}^{N-1} \frac{\partial n}{\partial r}\right)_{j} L_{j i},
\end{aligned}
$$

where $L_{j i} \equiv x_{j} \int \frac{\sqrt{r_{i+1}-x_{j}}}{\sqrt{r_{i}-x_{j}}}\left[(d y) / \sqrt{x_{j}^{2}+y^{2}}\right]$ and $(j=1,2, \ldots, N)$. Equation (9) can be expressed in a matrix form as

$$
\bar{C}=\frac{2 f_{2}}{a n_{\infty}}=\overline{\partial n} \frac{\overline{\partial n}}{\partial r},
$$

being the geometric coefficient matrix $\overline{\bar{L}}$ an upper diagonal matrix. By inverting $\overline{\bar{L}}$ the Eq. (10) can be easily solved to obtain the $\partial n / \partial r$ values at each annulus (in particular, note that $\partial n / \partial r)_{r=0}=0$ because of the problem symmetry). Integrating the obtained $\partial n / \partial r$ profile in the radial direction (with $n=n_{\infty}$ at the outer border of what) and using the refractive index given by the Eq. (4), the $T$ radial profile can be derived.

It is worth noting that the knowledge of the arc pressure is essential for the application of the presented procedure. This is the reason why in this type of arcs this method can be applied only to arc sections located below the characteristic shock front that appears close to the nozzle exit, where the arc pressure can be considered to reach the atmospheric value. $^{3}$

In Fig. 6 the obtained profile of the plasma temperature corresponding to an axial position located at $z=3.5 \mathrm{~mm}$ from the nozzle exit, is presented. The uncertainty in the plasma temperature profile is mainly due to the uncertainty in the $a$ value $( \pm 2.5 \%)$, and the corresponding $T$ profiles derived from the upper and lower $a$ values are also shown in Fig. 6 with gray (thin) lines. As it can be seen, the plasma temperature shows a monotonic decrease from the arc center (with a peak value $\approx 15 \mathrm{~K}$ ) up to the unperturbed region 


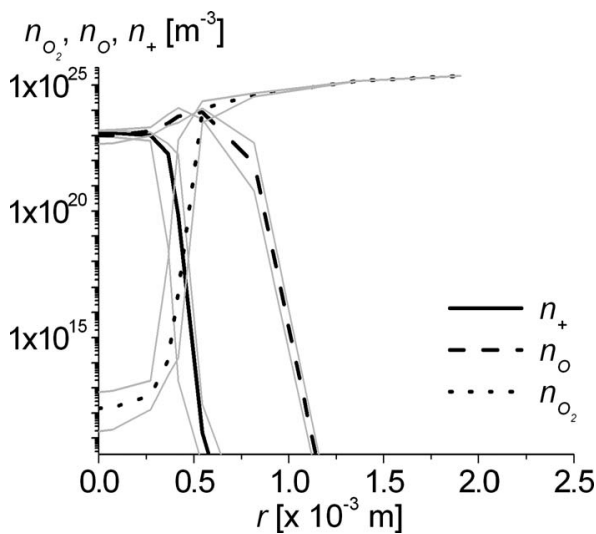

FIG. 7. Density profiles of the different plasma species corresponding to the temperature profile showed in Fig. 6. The gray thin lines indicate in each density profile the limits of its uncertainty range.

(with a gas temperature $\approx$ ambient temperature) at $\approx 2.2 \mathrm{~mm}$ from the arc axis. For comparative purposes, the plasma temperature radial profile previously obtained by the authors using an electrostatic probe technique ${ }^{16-18}$ is also shown in Fig. 6. As it can be seen, a relatively good agreement between both profiles is achieved, in spite of the fact that they were obtained using quite different plasma diagnostic techniques. However, the quantitative schlieren technique, due to its great sensibility, can provide accurate values of temperature in a wider range of radius values, from the arc axis to unperturbed zone). The radial range covered by the schlieren technique is also larger than that covered by spectroscopic techniques (the most employed diagnostic in cutting arcs) which only can provide the plasma temperature in the hotter region of the $\operatorname{arc}(T \geq 10 \mathrm{~K}, r \leq 0.3 \mathrm{~mm})$.

The radial profiles of the ionized oxygen $\left(n_{+}\right)$, atomic oxygen $\left(n_{\mathrm{O}}\right)$, and molecular oxygen $\left(n_{\mathrm{O}_{2}}\right)$ densities, are, respectively, shown in Fig. 7 with its corresponding uncertainty ranges (in gray, thin lines) derived from the uncertainty in the $T$ values. As quoted before, the densities were obtained from the temperature profile showed in Fig. 6 by using the state and Saha equations (for dissociation and ionization) at a plasma pressure of $0.1 \mathrm{MPa}$. As expected, the plasma is concentrated in the high temperature region $(T$ $>10 \mathrm{~K}$ ) within a radius of $\approx 0.3 \mathrm{~mm}$. On the other hand, $n_{O}$ increases continuously from the arc center (where the ionization degree is about $50 \%$ ) to $\approx 0.5 \mathrm{~mm}$ (where $n_{\mathrm{O}_{2}}$ becomes important) and then abruptly decrease at the periphery.

\section{CONCLUSIONS}

In this work, plasma temperature and radial density profiles of the plasma species in a high energy density cutting arc have been obtained by using a quantitative schlieren technique. To our knowledge, it is the first time that such analysis is reported for cutting torches, allowing the determination of the quoted quantities in a wide range of radial distances (from the arc axis to the unperturbed surrounding medium). The obtained temperature values are in good agreement with those values obtained by the authors on the same torch using the Langmuir probes technique.

\section{ACKNOWLEDGMENTS}

This work was supported by grants from the Universidad de Buenos Aires (Grant No. PID X111), CONICET (Grant No. PIP 5378), and UTN (Grant No. PID Z 012). One of the authors (L.P.) is grateful to the Fundación YPF. Also, B.M. is grateful to the Universidad Tecnológica Nacional. H.K. and G.A are members of the CONICET.

${ }^{1}$ V. A. Nemchinsky and W. S. Severance, J. Phys. D: Appl. Phys. 39, R423 (2006).

${ }^{2}$ L. Girard, Ph. Teulet, M. Razafinimanana, A. Gleizes, F. Camy-Peyret, E. Baillot, and F. Richard, J. Phys. D: Appl. Phys. 39, 1543 (2006).

${ }^{3}$ P. Freton, J. J. Gonzalez, A. Gleizes, F. Camy Peyret, G. Caillibotte, and M. Delzenne, J. Phys. D: Appl. Phys. 35, 115 (2002).

${ }^{4}$ P. Freton, J. J. Gonzalez, F. Camy Peyret, and A. Gleizes, J. Phys. D: Appl. Phys. 36, 1269 (2003).

${ }^{5}$ G. S. Settles, Schlieren and Shadowgraph Techniques (Springer, New York, 2001).

${ }^{6} \mathrm{C}$. Alvarez-Herrera, D. Moreno-Hernández, and G. Barrientos-García, J. Opt. A, Pure Appl. Opt. 10, 104014 (2008).

${ }^{7}$ E. Arnaud, E. Memin, R. Sosa, and G. Artana, Lect. Notes Comput. Sci. 3951, 198 (2006).

${ }^{8}$ V. Colombo, A. Concetti, E. Ghedini, S. Dallavalle, and M. Vancini, Plasma Sources Sci. Technol. 18, 023001 (2009).

${ }^{9}$ B. L. Bemis and G. S. Settles, Proceedings of the Eighth International Symposium on Flow Visualization, Sorrento, Italy, September 1998 (unpublished).

${ }^{10}$ B. L. Bemis and G. S. Settles, IEEE Trans. Plasma Sci. 27, 44 (1999).

${ }^{11}$ L. Prevosto, H. Kelly, and B. Mancinelli, J. Appl. Phys. 105, 013309 (2009).

${ }^{12}$ K. P. Hinz, J. Phys. D 19, 2381 (1986).

${ }^{13}$ Y. P. Raizer, Gas Discharge Physics (Springer, Berlin, 1991).

${ }^{14}$ M. Boulos, P. Fauchais, and E. Pfender, Thermal Plasmas, Fundamentals, and Applications (Plenum, New York, 1994), Vol. 1, Chap. 4.

${ }^{15}$ W. Lochte-Holtgreven, Plasma Diagnostics (AIP, New York, 1995), Chap. 3.

${ }^{16}$ L. Prevosto, H. Kelly, and B. Mancinelli, IEEE Trans. Plasma Sci. 36, 263 (2008).

${ }^{17}$ L. Prevosto, H. Kelly, and B. Mancinelli, IEEE Trans. Plasma Sci. 36, 271 (2008).

${ }^{18}$ L. Prevosto, H. Kelly, and B. Mancinelli, IEEE Trans. Plasma Sci. 37, 1092 (2009). 